\title{
Admission hyperglycemia and the no- reflow phenomenon in patients with acute myocardial infarction
}

\begin{abstract}
Background: Coronary artery disease (CAD) is the single most common cause of death in the developed world, responsible for about one in every six deaths; 15.5 million person's $>20$ years of age in the USA have coronary heart disease (CHD). No-reflow phenomenon defined classically as the lack of myocardial perfusion despite opening up the epicardial vessel in the setting of $\mathrm{PPCl}$ occurring in $10 \%$ of cases of $\mathrm{PPCl}$ and associated with increase in 30 days mortality caused by multiple factors that lead to micro vascular obstruction and endothelial disruption by different mechanism like atherothrombotic embolization, ischemic injury, reperfusion injury and susceptibility of coronary microcirculation to injury.
\end{abstract}

Objective: The aim of the study to is to investigate the correlation between admission blood glucose level and the no-reflow phenomenon in patients with AMI undergoing Primary PCl.

Patients and methods: This study was Cross-sectional study which was conducted on patients presenting with acute ST segment elevation myocardial infarction who underwent primary PCl. The study included 280 patients presented with acute MI within less than 24 hours from onset of symptoms. All study population will undergo full medical history, full clinical examination, Blood sample and chemistry, electrocardiography, transthoracic echocardiography and coronary angiography.

Results: Admission hyperglycemia with AMI among AMI patients was independent predictor for incidence of no-reflow. Diabetics after classified into hyperglycemic and non-hyperglycemic, hyperglycemic in diabetics has significant high no-reflow rate compared to non-diabetics.

Conclusion: We established an association between hyperglycemia and the no-reflow phenomenon.

Keywords: Acute myocardial infarction no-reflow phenomenen Admission acuteh

Introduction: Coronary artery disease (CAD) is the single most common cause of death in the developed world, responsible for about one in every six deaths [1]. Mortality from cardiovascular disease, in general, is estimated to reach 23.4 million in 2030 [2]. More than 4 million Europeans die of Cardiovascular disease (CVD) every year, of whom approximately half due to CAD [3].

The Heart Disease and Stroke statistics update of the American Heart Association (AHA) has recently reported that 15.5 million person's $>20$ years of age in the USA have coronary heart disease (CHD) [4].

The acute coronary syndrome (ACS) include myocardial infraction with ST segment elevation (STEMI), non-ST elevation myocardial infarction (NSTEMI) that is diagnosed by enzyme rise and unstable angina when there are new or worsening symptoms of ischaemi, and ischaemic ECG changes with normal biomarkers [5].

Diagnostic tests for the detection of myocardial ischaemia are most
Interventional Cardiology

\author{
Omar F Tawfik*, Mosbah T \\ Hassanin, Mohy Eldin Aboelfetoh \\ El-Deeb and Mohamed Emara \\ Department of Cardiology, Faculty of \\ Medicine, Zagazig University, Zagazig, Egypt \\ *Author for correspondence: \\ Omar F Tawfik, Department of Cardiology, \\ Faculty of Medicine, Zagazig University, \\ Zagazig, Egypt, \\ Email:dr_Omar_Farid@yahoo.com
}

useful in patients with an intermediate pretest probability of CAD and are recommended for all patients with an intermediate or high probability of CAD. This include exercise treadmill test, stress echocardiography, magnetic resonance imaging, coronary artery computed tomography (CCT), coronary angiography. Hyperglycemia can be observed in patients with acute myocardial infarction (AMI), irrespective of a history of diabetes mellitus (DM) and is associated with increased mortality after AMI. The decrease in blood glucose, by an insulin-glucose infusion, during the first 24 hours after AMI decreases mortality in patients with DM [6].

Acute hyperglycemia is common in patients with STEMI even in the absence of a history of type 2 DM. Hyperglycemia is encountered in up to $50 \%$ of all STEMI patients, whereas previously diagnosed DM is present in only $20 \%$ to $25 \%$ of STEMI patients [7]. The prevalence of type 2 DM or impaired glucose tolerance may be as high as $65 \%$ in MI patients without prior DM when oral glucose tolerance testing is performed [8]. Elevated plasma glucose and glycated hemoglobin levels on admission are independent prognosticators of both in-hospital and long-term outcome regardless of diabetic status $[9,10]$. For every $18 \mathrm{mg} / \mathrm{dL}$ increase in glucose level, there is a $4 \%$ increase in mortality in non-diabetic subjects [11].

When admission glucose level exceeds $200 \mathrm{mg} / \mathrm{dL}$, mortality is similar in nonDM and DM subjects with MI. Admission glucose has been identified as a major independent predictor of both in-hospital congestive heart failure and mortality in STEMI [12].

The increased mortality in patients with hyperglycemia might be explained by a larger infarct size, a high incidence of congestive heart failure, and cardiogenic shock [13].

However, the underlying mechanisms of these deleterious effects of hyperglycemia are not well understood. Impaired microvascular function, or the no-reflow phenomenon, determines functional and clinical outcomes after AMI [14].

No-reflow phenomenon defined classically as the lack of myocardial perfusion despite opening up the epicardial vessel in the setting of PPCI occurring in $10 \%$ of cases of PPCl and associated with increase in 30 days mortality caused by multiple factors that lead to microvascular obstruction and endothelial disruption by different mechanism like atherothrombotic embolization, ischemic injury, reperfusion injury and susceptibility of coronary microcirculation to injury [15].

The aim of the study to is to investigate the correlation between admission blood glucose level and the no-reflow phenomenon in patients with AMI undergoing Primary PCI. 


\section{Patients and Methods}

This study was Cross-sectional study which was conducted on patients presenting with acute ST segment elevation myocardial infarction who underwent primary PCI.

\section{Inclusion criteria}

All patients with the definite diagnosis of acute myocardial infarction who underwent primary PCI. Acute myocardial infarction according to the 4th universal definition of myocardial infarction as: Typical rise of biochemical markers of myocardial necrosis with at least one of the following [14]:

i. Ischemic symptoms as chest pain within less than 24 hours.

ii. ECG changes indicative of ischemia: New ST elevation at the J-point in two contiguous leads with $>2.5$ big square in male less than 40 years old or $>2$ big square in men more than 40 years old or $>1.5$ big square in women in leads $\mathrm{v} 2-\mathrm{v} 3 \mathrm{and} /$ or 1 big square in other leads.

iii. New onset LBBB OR ST elevation in RT leads v3-v4.R or in posterior leads v7-v9 or ST depression in v1-v3>0.5 big square with upright T-wave.

iv. Imaging evidence of new loss of viable myocardium or new regional wall motion abnormality.

\section{Exclusion criteria}

i. Cardiogenic shock: patient presented with Killip class IV excluded from our study as there is no afterload so no coronary perfusion present and no myocardial perfusion.

ii. Unsuccessful PCI (e.g: Allergy to contrast).

iii. Missed MI with onset of symptoms exceeds 24 hours as it already increase risk of no-reflow that will influence our result.

iv. Advanced liver or kidney diseases.

v. Patients refusing consent for enrollment.

\section{Methods}

1. Full history taking.

2. Clinical examination with special emphasis on ABP, heart rate (on presentation and at discharge) and Killip class on presentation.

3. Twelve lead surface ECG.

4. Laboratory investigations including random blood glucose level on admission, serum troponin CK and CK-MB (ng/ $\mathrm{ml})$ levels, serum creatinine and HBA1C.

5. Coronary angiography and primary PCI.

6. Transthoracic echocardiography.

\section{TIMI flow prior to and after procedure}

- Grade 0 (no perfusion): There is no antegrade flow beyond the point of occlusion.

- $\quad$ Grade 1 (penetration without perfusion): The contrast material passes beyond the area of obstruction but "hangs up" and fails to opacify the entire coronary bed distal to the obstruction for the duration of the cine-angiographic filming sequence.

- $\quad$ Grade 2 (partial perfusion): The contrast material passes across the obstruction and opacifies the coronary bed distal to the obstruction. However, the rate of entry of contrast material into the vessel distal to the obstruction or its rate of clearance from the distal bed (or both) is perceptibly slower than its entry into or clearance from comparable areas not perfused by the previously occluded vessel.

- $\quad$ Grade 3 (complete perfusion): Antegrade flow into the bed distal to the obstruction occurs as promptly and as rapid as clearance from an uninvolved bed in the same vessel or the opposite artery.

- Myocardial blush grade will be evaluated as:

- Grade 0: No myocardial contrast density.

- Grade 1: Minimal myocardial contrast density.

- $\quad$ Grade 2: Moderate myocardial contrast density that is less than the territory perfused by any non-infarct-related coronary artery.

- $\quad$ Grade 3: Normal myocardial contrast density compared to any non-infarct related coronary artery territory.

\section{Medications}

Medical treatment was given to subjects according to hospital protocol and the following were stressed upon. During hospitalization, acetyl salicylic acid, clopidogrel, anticoagulant, statins, ACEIs, nitrates and beta blockers were given to the patient if not contraindicated.

\section{Statistical analysis}

Data were analyzed using Statistical Program for Social Science (SPSS) version 25.0 for windows (SPSS Inc., Chicago, IL, USA) and NCSS 12.0 for windows (NCSS LCC., Kaysville, UT, USA).

Quantitative data were expressed as mean \pm standard deviation (SD). Median and inter-quartile range (IQR) were also calculated for quantitative data. Qualitative data were expressed as frequency and percentage.

The following tests were done: independent-samples t-test of significance, Mann Whitney U test, Chi-square (X2) test, Fisher Exact test, the "Linear-by-Linear" test.

Multivariate regression analysis is used when we want to predict the value of a variable based on the value of two or more other 
variables. The variable we want to predict is called the dependent variable. The variables we are using to predict the value of the dependent variable are called the independent variables (or sometimes, the predictor, explanatory or regressor variables).

Receiver operating characteristic (ROC) curve analysis was used to identify optimal cut-off values. Area Under Curve (AUC) was also calculated. The optimal cutoff point was established at point of maximum accuracy; p-value $<0.05$ was considered significant, $\mathrm{p}$-value $<0.001$ was considered as highly significant and $\mathrm{p}$-value $>0.05$ was considered insignificant.

\section{Results}

In Table 1, total cases were 280 patients with ACS 205 were male (73.2\%) and female was 75 (26.8) with mean age (55.0 \pm 9.7$)$ and median 55 (49-61). Risk factors counted as follow:

- $\quad 139$ (49.6\%) had HTN and 110 (39.3\%) Was DM.

- $\quad 152(54.3 \%)$ had special habit like smoking.

- $\quad 67(23.9 \%)$ was dyslipidemic and 57 (20.4\%) had family history of CAD and 54 (19.3\%) were known ischemic before.

Table 1: Demographic data of the whole study population.

\begin{tabular}{|c|c|}
\hline Demographic data & All patients \\
\hline Count (\%) & $280(100 \%)$ \\
\hline \multicolumn{2}{|l|}{ Gender } \\
\hline Male & 205 (73.2\%) \\
\hline Female & 75 (26.8\%) \\
\hline \multicolumn{2}{|l|}{ Age (years) } \\
\hline Mean \pm SD & $55.0 \pm 9.7$ \\
\hline Median (IQR) & $55(49-61)$ \\
\hline \multicolumn{2}{|l|}{ Risk factors } \\
\hline HTN & 139 (49.6\%) \\
\hline DM & 110 (39.3\%) \\
\hline Smoking & $152(54.3 \%)$ \\
\hline Dyslipidemia & 67 (23.9\%) \\
\hline Family history & 57 (20.4\%) \\
\hline Known CAD & 54 (19.3\%) \\
\hline
\end{tabular}

In Table 2, systolic blood pressure of total cases measured with Mean \pm SD (120.8 \pm 17.2) with median 120 (110-130) but Heart rate Mean \pm SD was $(91.4 \pm 13.9)$ with median 90 (84-100).

Onset of chest pain to admission time was Mean \pm SD $(8.5 \pm 5.8)$ with median 7 (4-12) so passed time 24 hours was excluded and Killip class IV (Cardiogenic shock) also excluded but Killip class III included with $40(14.3 \%)$ percentage and 6 (2.1\%) develop DKA of total cases.

\begin{tabular}{|c|c|}
\hline \multicolumn{2}{|c|}{ Table 2: Clinical data of the whole study population. } \\
\hline Clinical data & All patients \\
\hline Count (\%) & $280(100 \%)$ \\
\hline SBP (mmHg) & $120.8 \pm 17.2$ \\
\hline Mean \pm SD & $120(110-130)$ \\
\hline Median (IQR) & \\
\hline HR (beat/min) & $91.4 \pm 13.9$ \\
\hline Mean \pm SD & $90(84-100)$ \\
\hline Median (IQR) & \\
\hline Chest pain duration (hours) & $8.5 \pm 5.8$ \\
\hline Mean \pm SD & $7(4-12)$ \\
\hline Median (IQR) & $40(14.3 \%)$ \\
\hline Killip class III & $6(2.1 \%)$ \\
\hline DKA &
\end{tabular}

Echocardiography was done to all patients and data collected show LVEDD $(\mathrm{cm})$ Mean \pm SD $(5.5 \pm 0.6)$ with Median 5.5 (5.0-5.9), LVESD $(\mathrm{cm})$ Mean \pm SD $(4.0 \pm 0.6)$ with Median 4.0 (3.6-4.4), Ejection fraction Mean \pm SD $(45.0 \pm 8.2)$ with Median 45 (40-50), Wall motion score index Mean \pm SD $(1.53 \pm 0.34)$ with Median 1.45 (1.25-1.75), LA diameter $(\mathrm{cm})$ Mean \pm SD $(3.9 \pm 0.5)$ with Median 3.9 (3.6-4.1) and Diastolic dysfunction incidence as 24 (8.6\%) without, 188 (67.1\%) had grade I, 58 (20.7\%) had Grade II and 10 (3.6\%) had Grade III (Table 3).

\begin{tabular}{|c|c|}
\hline Echocardiographic data & All patients \\
\hline Count (\%) & 280 (100\%) \\
\hline \multicolumn{2}{|l|}{ LVEDD $(\mathrm{cm})$} \\
\hline Mean \pm SD & $5.5 \pm 0.6$ \\
\hline Median (IQR) & $5.5(5.0-5.9)$ \\
\hline \multicolumn{2}{|l|}{ LVESD $(\mathrm{cm})$} \\
\hline Mean \pm SD & $4.0 \pm 0.6$ \\
\hline Median (IQR) & $4.0(3.6-4.4)$ \\
\hline \multicolumn{2}{|l|}{ EF (\%) } \\
\hline Mean \pm SD & $45.0 \pm 8.2$ \\
\hline Median (IQR) & $45(40-50)$ \\
\hline \multicolumn{2}{|l|}{ WMSI } \\
\hline Mean \pm SD & $1.53 \pm 0.34$ \\
\hline Median (IQR) & $1.45(1.25-1.75)$ \\
\hline \multicolumn{2}{|l|}{ LA diameter $(\mathrm{cm})$} \\
\hline Mean \pm SD & $3.9 \pm 0.5$ \\
\hline Median (IQR) & $3.9(3.6-4.1)$ \\
\hline \multicolumn{2}{|l|}{ Diastolic dysfunction } \\
\hline No diastolic dysfunction & $24(8.6 \%)$ \\
\hline Grade I & $188(67.1 \%)$ \\
\hline Grade II & $58(20.7 \%)$ \\
\hline Grade III & $10(3.6 \%)$ \\
\hline
\end{tabular}

In Table 4, all patients undergoing Primary PCI showing 205 (73.2\%) had culprit artery as Left Anterior Descending artery 
(LAD), 19 (6.8\%) had culprit artery as Left Circimflux artery (LCX)and 56 (20\%) had Right Coronary artery (RCA) as culprit vessel.

\begin{tabular}{|c|c|c|}
\hline \multicolumn{3}{|c|}{$\begin{array}{r}\text { Table 4: CA data and in-hospital mo } \\
\text { population }\end{array}$} \\
\hline \multicolumn{2}{|c|}{ CA data and in-hospital outcome } & All patients \\
\hline \multicolumn{2}{|c|}{ Count (\%) } & $280(100 \%)$ \\
\hline \multicolumn{3}{|l|}{ Culprit artery } \\
\hline \multicolumn{2}{|l|}{ LAD } & $205-73.2 \%$ \\
\hline \multicolumn{2}{|l|}{ LCX } & $19-6.8 \%$ \\
\hline \multicolumn{2}{|l|}{ RCA } & $56(20 \%)$ \\
\hline \multicolumn{3}{|c|}{ TIMI flow post PCI } \\
\hline TIMI 0 & \multirow{2}{*}{$\begin{array}{c}\text { No-reflow } \\
-34.30 \%\end{array}$} & $38(13.6 \%)$ \\
\hline TIMI 1 & & $58(20.7 \%)$ \\
\hline TIMI 2 & \multirow{3}{*}{$\begin{array}{c}\text { Reflow } \\
-65.70 \% \\
\end{array}$} & $22-7.9 \%$ \\
\hline TIMI 3 & & $162-57.8 \%$ \\
\hline In-hospital mortality & & $36(12.9 \%)$ \\
\hline
\end{tabular}

No-reflow (TIMI 0 and I) percentage was (34.3\%) as $38(13.6 \%)$ develop TIMI 0 and 58 (20.7\%) develop TIMI 1 but reflow (TIMI II and III) was (65.7\%) as $22(7.9 \%)$ develop TIMI II and 162 (57.8\%) develop TIMI III with In-hospital mortality 36 (12.9\%).

Patients divided to 2 groups according to admission glucose level with cutoff (200 mg-dl) to hyperglycemic group (148) and Euglycemic group (132). There is high significant difference in Diabetic patient p-value $<0.001$ (HS) (Table 5).

\begin{tabular}{|c|c|c|c|c|}
\hline $\begin{array}{c}\text { Demographic } \\
\text { data }\end{array}$ & Euglycemic & Hyperglycemic & \multirow[t]{2}{*}{ Test } & \multirow{2}{*}{$\begin{array}{c}\text { p-value } \\
\text { (Sig) }\end{array}$} \\
\hline Count & 132 & 148 & & \\
\hline \multicolumn{5}{|l|}{ Gender } \\
\hline Male & 101 (76.5\%) & 104 (70.3\%) & \multirow{2}{*}{$1.387 \neq$} & \multirow{2}{*}{0.239 (NS) } \\
\hline Female & 31 (23.5\%) & 44 (29.7\%) & & \\
\hline Mean \pm SD & $54.4 \pm 9.9$ & $55.6 \pm 9.6$ & $-1.070 *$ & 0.285 (NS) \\
\hline \multicolumn{5}{|l|}{ Risk factors } \\
\hline HTN & 65 (49.2\%) & 74 (50\%) & $0.016 \neq$ & 0.899 (NS) \\
\hline $\mathrm{DM}$ & $34(25.8 \%)$ & 76 (51.4\%) & $19.161 \neq$ & $<0.001$ \\
\hline Smoking & 78 (59.1\%) & $74(50 \%)$ & $2.324 \neq$ & 0.127 (NS) \\
\hline Dyslipidemia & $28(21.2 \%)$ & 39 (26.4\%) & $1.012 \neq$ & 0.314 (NS) \\
\hline Family history & $23(17.4 \%)$ & $34(23 \%)$ & $1.325 \neq$ & 0.250 (NS) \\
\hline Known CAD & 24 (18.2\%) & 30 (20.3\%) & $0.195 \neq$ & 0.658 (NS) \\
\hline
\end{tabular}

Killip class III occurred with median 12 (9.1\%) and 28 (18.9\%) with significance p-value 0.019 (S). DKA occurred with median 0 $(0 \%)$ and $6(4.1 \%)$ with significance p-value $6(4.1 \%) 0.031(\mathrm{~S})$ (Table 6).

In Table 7, max CKMB measured between the 2 groups with Median 105 (60-198) and 121.5 (74-208) and there was significant difference with p-value $0.033(\mathrm{~S})$. Max CK total measured between the 2 groups with Median 1280 (848-1616) and 1500 (940-2634) and there was significant difference with p-value $0.012(\mathrm{~S})$.
Admission $\mathrm{Bl}$ glucose level measured between the 2 groups with Median 140 (120-170) and 300 (255-340) and there was significant difference with p-value $0.026(\mathrm{~S})$. Createnine measured between the 2 groups with Median 0.9 (0.7-1.1) and $1.0(0.8-1.2)$ and there was significant difference with p-value $0.026(S)$.

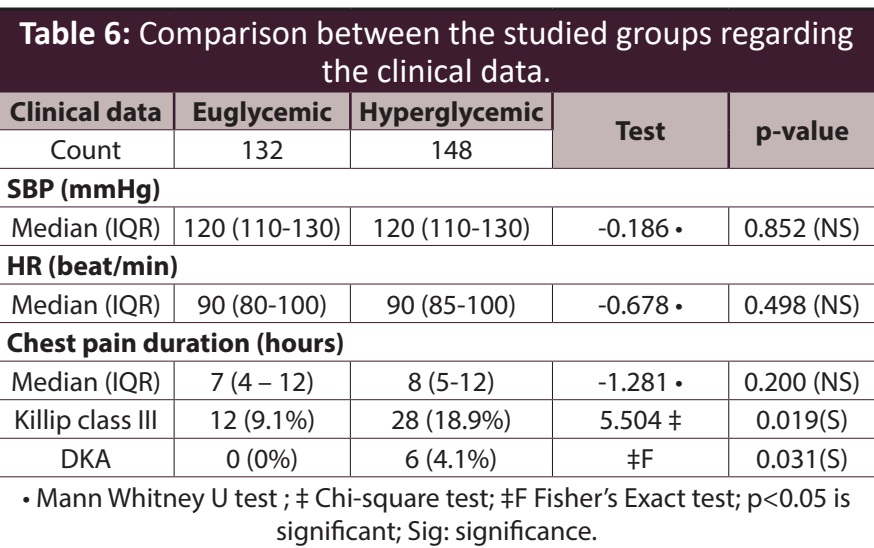



Final TIMI Flow had high significant p-value $<0.001$ (HS) between 2 groups as TIMI O 6 (4.5\%) vs. 32 (21.6\%), TIMI $116(12.1 \%)$ vs. $42(28.4 \%)$, TIMI $212(9.1 \%)$ vs. $10(6.8 \%)$ and TIMI 398 (74.2\%) vs. $64(43.2 \%)$ respectively $22 / 110$ (16.7\%-83.3\%) and 74/74 (50\%-50\%). In-hospital mortality had significant p-value $0.004(\mathrm{~S})$ as $9(6.8 \%)$ and $27(18.2 \%)$ respectively (Table 8$)$.

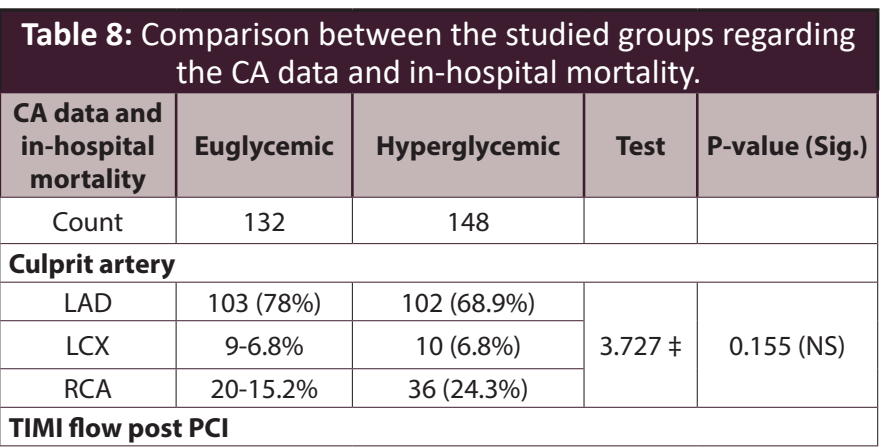




\begin{tabular}{|c|c|c|c|c|}
\hline TIMI 0 & $6-4.5 \%$ & $32(21.6 \%)$ & \multirow{4}{*}{$\begin{array}{c}34.943 \\
\neq\end{array}$} & \multirow{4}{*}{$<0.001(\mathrm{HS})$} \\
\hline TIMI 1 & $16-12.1 \%$ & 42 (28.4\%) & & \\
\hline TIMI 2 & 12 (9.1\%) & $10(6.8 \%)$ & & \\
\hline TIMI 3 & $98-74.2 \%$ & $64(43.2 \%)$ & & \\
\hline \multicolumn{5}{|c|}{ Final flow post $\mathrm{PCI}$} \\
\hline No-reflow & $22-16.7 \%$ & $74(50 \%)$ & \multirow{2}{*}{$\begin{array}{c}34.408 \\
\neq\end{array}$} & \multirow{2}{*}{$<0.001(\mathrm{HS})$} \\
\hline Reflow & $110(83.3 \%)$ & $74(50 \%)$ & & \\
\hline $\begin{array}{l}\text { In-hospital } \\
\text { mortality }\end{array}$ & $9-6.8 \%$ & 27 (18.2\%) & $8.129 \neq$ & $0.004(\mathrm{~S})$ \\
\hline
\end{tabular}

Significant $\mathrm{p}$-value found in univariate factors for incidence of no-reflow as follow age (years) 0.003, DM 0.033, CK-MB $(\mathrm{U} / \mathrm{L})<0.001$, RBS $(\mathrm{mg} / \mathrm{dL})<0.001$ and $S$. Creatinine $(\mathrm{mg} / \mathrm{dL})$ 0.008 (Table 9).

\begin{tabular}{|c|c|c|c|c|}
\hline \multirow{2}{*}{$\begin{array}{l}\text { CA data and } \\
\text { in-hospital } \\
\text { mortality }\end{array}$} & \multirow{2}{*}{ Euglycemic } & \multicolumn{2}{|c|}{$\begin{array}{l}\text { 95\% Confidence Interval } \\
\text { for OR }\end{array}$} & \multirow{2}{*}{$\begin{array}{l}\text { P-value } \\
\text { (Sig.) }\end{array}$} \\
\hline & & $\begin{array}{l}\text { Lower } \\
\text { Bound }\end{array}$ & $\begin{array}{l}\text { Upper } \\
\text { Bound }\end{array}$ & \\
\hline Male gender & 0.77 & 0.445 & 1.333 & 0.351 \\
\hline Age (years) & 1.042 & 1.014 & 1.07 & 0.003 \\
\hline HTN & 1.497 & 0.911 & 2.461 & 0.111 \\
\hline DM & 1.725 & 1.044 & 2.851 & 0.033 \\
\hline Smoking & 0.635 & 0.386 & 1.043 & 0.073 \\
\hline Dyslipidemia & 0.84 & 0.467 & 1.512 & 0.561 \\
\hline $\begin{array}{l}\text { Family } \\
\text { history }\end{array}$ & 0.858 & 0.46 & 1.599 & 0.63 \\
\hline Known CAD & 1.412 & 0.767 & 2.598 & 0.267 \\
\hline $\mathrm{SBP}(\mathrm{mmHg})$ & 0.993 & 0.978 & 1.007 & 0.33 \\
\hline $\begin{array}{l}\text { HR (beat/ } \\
\text { min) }\end{array}$ & 1.008 & 0.991 & 1.026 & 0.365 \\
\hline $\begin{array}{l}\text { Chest pain } \\
\text { duration } \\
\text { (hours) }\end{array}$ & 1.024 & 0.982 & 1.068 & 0.27 \\
\hline $\begin{array}{c}\text { Killip class III } \\
\text { and IV }\end{array}$ & 1.333 & 0.671 & 2.651 & 0.412 \\
\hline DKA & 0.957 & 0.172 & 5.323 & 0.96 \\
\hline CK-MB (U/L) & 1.004 & 1.002 & 1.006 & $<0.001$ \\
\hline CK-total (U/L) & 1 & 1 & 1 & 0.165 \\
\hline $\mathrm{RBS}(\mathrm{mg} / \mathrm{dL})$ & 1.013 & 1.009 & 1.016 & \\
\hline $\begin{array}{l}\text { S. Creatinine } \\
(\mathrm{mg} / \mathrm{dL})\end{array}$ & 3.616 & 1.392 & 9.393 & \\
\hline $\begin{array}{c}\text { LAD as a } \\
\text { culprit artery }\end{array}$ & 1.484 & 0.832 & 2.646 & 0.182 \\
\hline
\end{tabular}

Multivariate regression analysis for incidence of no-reflow show significant p-value in CK-MB (U/L) 0.009 and RBS (mg/ $\mathrm{dL})<0.001$ (Figure 1).

Comparison between CK-MB $\geq 116 \mathrm{U} / \mathrm{L}$ and $\mathrm{RBS} \geq 241 \mathrm{mg} /$ $\mathrm{dL}$ as a cut off for prediction of no-reflow as follow Sensitivity 60.4\% (49.9-70.3) and 72.9\% (62.9-81.5), Specificity 56.5\% (49.0-63.8) and 76.1\% (69.3-82.1), Positive Predictive Value 42\% (33.7-50.7) and 61.4\% (51.8-70.4), Negative Predictive Value $73.2 \%(65.2-80.3)$ and $84.3 \%(77.9-89.5)$, Accuracy $57.9 \%$
(51.8-63.7) and 75\%(69.5-80.0), Area Under Receiver Operating Characteristic curve 0.636 (0.561-0.701) and 0.808 (0.748-0.855) and p-value $<0.001$ (HS) and $<0.001$ (HS) respectively (Table 10).

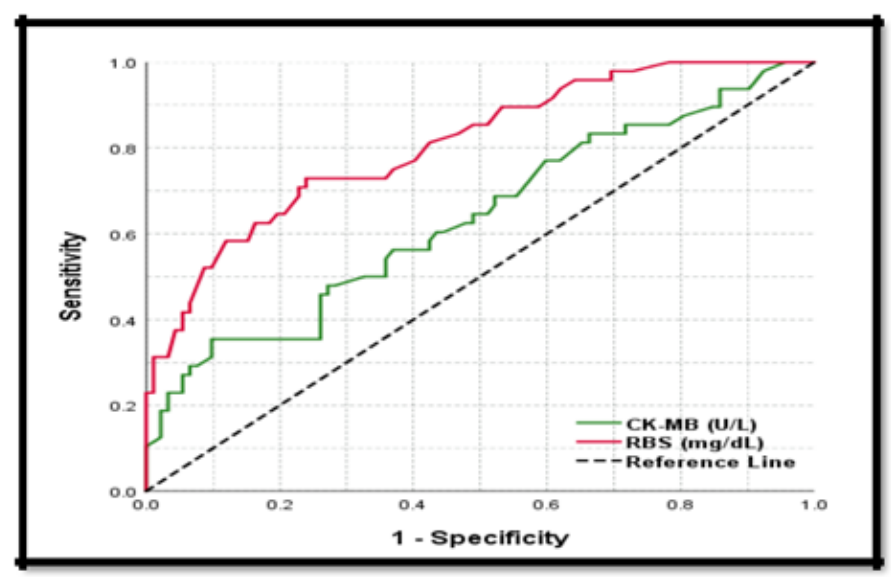

Figure 1: ROC curve analysis for predictors of no-reflow.

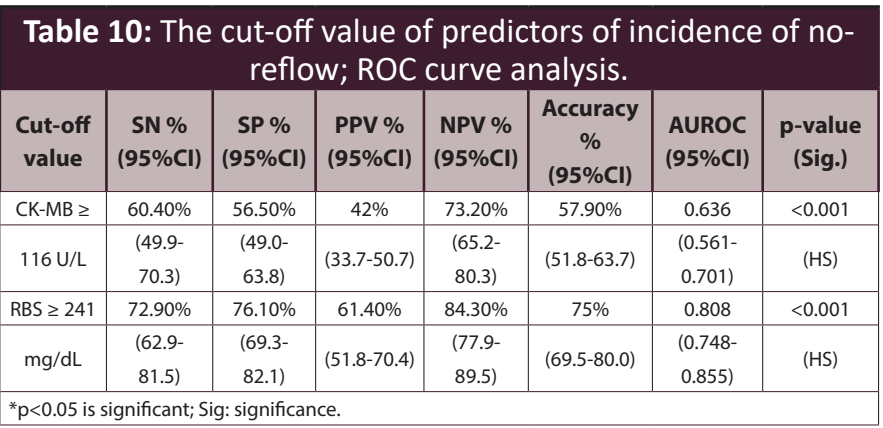

Relation between cases as 4 groups as seen above show significant $<0.001$ (HS) show that incidence of no-reflow increase with hyperglycemia (Table 11).

Table 11: Comparison between different DM and glycemic statuses regarding incidence of no-reflow.

\begin{tabular}{|c|c|c|c|c|c|c|}
\hline $\begin{array}{c}\text { Upper } \\
\text { Bound }\end{array}$ & $\begin{array}{c}\text { DM } \\
\text { Hyperg- } \\
\text { lycemic }\end{array}$ & $\begin{array}{c}\text { DM } \\
\text { Eugly- } \\
\text { cemic }\end{array}$ & $\begin{array}{c}\text { Non-DM } \\
\text { Hyperg- } \\
\text { lycemic }\end{array}$ & $\begin{array}{c}\text { Non-DM } \\
\text { Eugly- } \\
\text { cemic }\end{array}$ & Test & $\begin{array}{c}\text { P-value } \\
\text { (Sig.) }\end{array}$ \\
\hline Count & 76 & 34 & 72 & 98 & & \\
\hline \multicolumn{7}{|l|}{ TIMI flow post PCI } \\
\begin{tabular}{|c|c|c|c|c|c|} 
No- \\
reflow
\end{tabular} & $40(52.6 \%)$ & $6(17.6 \%)$ & $34(47.2 \%)$ & $\begin{array}{c}16 \\
(16.3 \%)\end{array}$ & $\begin{array}{c}34.908 \\
\neq\end{array}$ & $\begin{array}{c}<0.001 \\
(\mathrm{HS})\end{array}$ \\
\hline Reflow & $36(47.4 \%)$ & $28(82.4 \%)$ & $38(52.8 \%)$ & $\begin{array}{c}82 \\
(83.7 \%)\end{array}$ & & \\
\hline
\end{tabular}

Case no. 1

- 69 years old male, smoker, not diabetic, hypertensive, smoker no family history of CAD.

- $\quad$ Presented to ER with reterosternal chest pain described as heaviness, radiated to left arm, within 12 hours after onset of 


\section{Research Article}

symptoms.

- $\quad$ By clinical examination: Blood pressure: 110/60 mmhg, HR: 75 bpm, Auscultation: Normal heart sound, no murmers with basal fine creps.

- $\quad$ ECG: showed ST elevation in leads v1 to v6 (Figure 2).

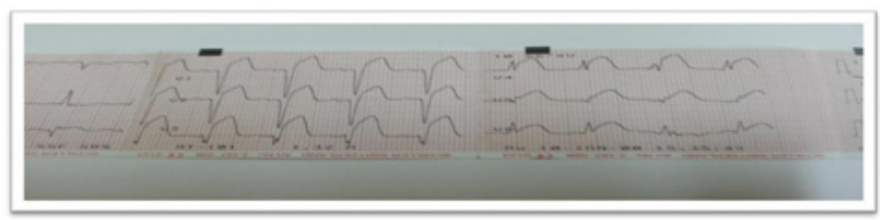

Figure 2: ECG: ST elevation for Case 1.

- Laboratory investigations: Admission blood glucose $330 \mathrm{mg} / \mathrm{dl}$,Troponin +ve, CKMB 185, CK 1370, Creatinine 1.3, Normal lipid file. Echocardiography: EF 35-40\%, resting wall motion abnormality in the form of severe hypokinesia on LAD Territory with D Dys G I, WMS index 1.9.

- $\quad$ Killip class II.

- $\quad$ PCI done with 1 DES and patient develop TIMI I to 0 as pictures shown.

- $\quad$ Patient develop No-reflow TIMI 0 improved to TIMI I after multiple adrenaline and nitrate intracoronary (Figure 3 ).
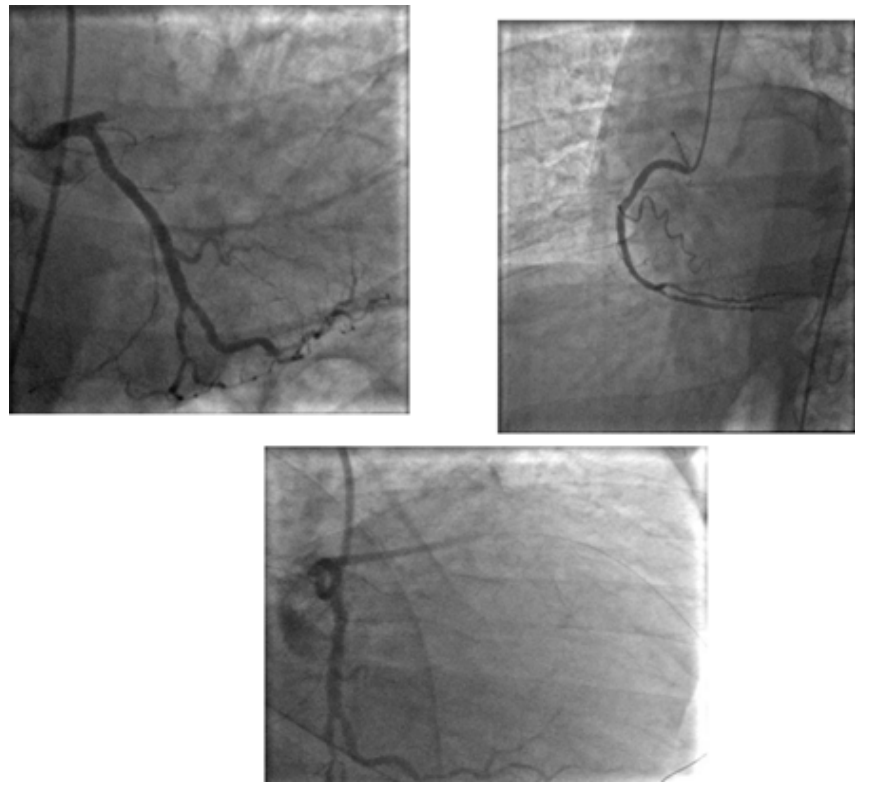

Figure 3: Patient develop No-reflow TIMI 0 improved to TIMI I after multiple adrenaline and nitrate intracoronary.

Case no. 2

- $\quad 56$ years old male, not diabetic, hypertensive, smoker no family history of CAD.

- $\quad$ Presented to ER with reterosternal chest pain described as heaviness, radiated to left arm, back, within 6 hours after onset of symptoms.

- $\quad$ By clinical examination: Blood pressure: 100/60 mmh, HR: 70 bpm, Auscultation: Normal heart sound, no murmers with clear chest.

- $\quad$ ECG: showed ST elevation in leads v1 to v6 (Figure 4).

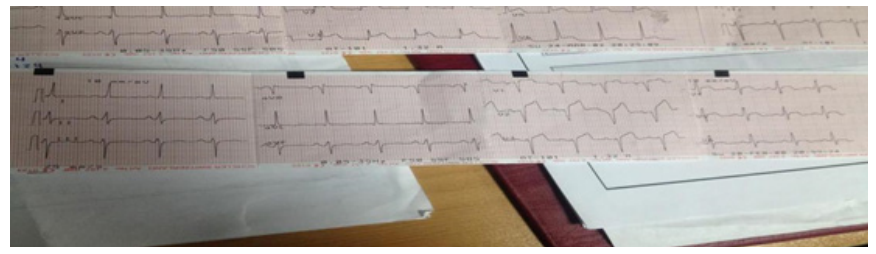

Figure 4: ECG: ST elevation for Case 2.

- Laboratory investigations: Admission blood glucose 155 mg/dl, Troponin +ve, max CKMB 250 and CK 2300, Creatinine 1.1, Normal lipid file. Echocardiography: EF 45\%, resting wall motion abnormality in the form of hypokinesia on LAD Territory and lat wall with D Dys G I, WMS index 1.6.

- $\quad$ Killip class I.

- $\quad$ PCI was done with 1 DES with TIMI I Flow that was improved to TIMI II Flow after intracoronary aggrestat and adrenaline due to heavy thrombus burden (Figure 5).
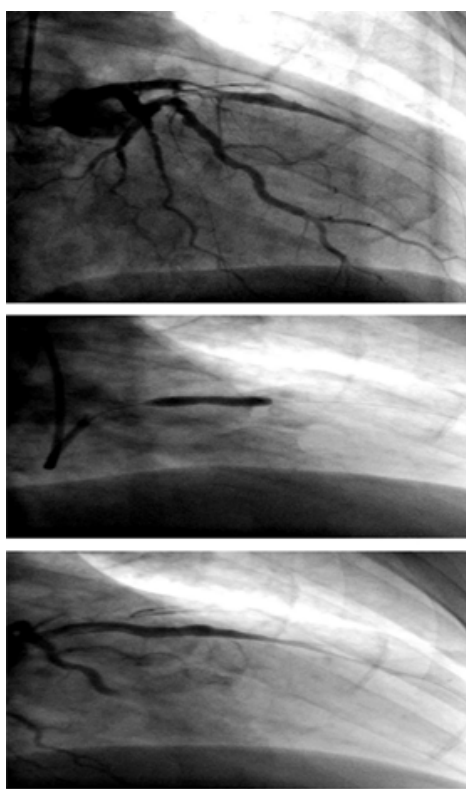

Figure 5: PCI done with 1 DES with TIMI I Flow that was improved to TIMI II Flow after intracoronary aggrestat and adrenaline due to heavy thrombus burden.

\section{Discussion}

Hyperglycemia can be seen in the course of acute MI irrelative to DM; and it is associated with increased mortality after MI. The relationship between "no-reflow" phenomenon and acute hyperglycemia can be explained by a lot of mechanisms. First, there 


\section{Research Article}

is an increase in the obstruction of capillary bed with leucocytes by increasing the levels of ICAM-1 or p-selectin. The accumulation of leucocytes in coronary capillary bed after coronary perfusion is higher in diabetic animal hearts when compared to non-diabetic animals. Leucocyte plugs in capillary bed are among the factors that contribute to the development of "no-reflow" phenomenon [16].

Moreover, hyperglycemia increases the occurrence of thrombus. The occurrence of micro thrombus is one of the key reasons of "noreflow" phenomenon. Finally, it is suggested that hyperglycemia is associated with reperfusion injury. In the heart of mouse, myocardial reperfusion is increased by hyperglycemia that causes the increment of the adhesion of leucocytes to capillary bed and production of free oxygen radicals [16].

Relation of acute hyperglycemia on admission and worse prognosis is higher in non-diabetic patients compared to diabetic patients $[17,18]$.

In Korea Acute Myocardial Infarction Registry (KAMIR), the relationship between hyperglycemia on admission and mortality was studied for AMI patients without history of diabetes. It shows that hyperglycemia on admission $\geq 200 \mathrm{mg} / \mathrm{dL}$ has significantly higher one year mortality rate compared to non-hyperglycemia on admission [18].

However, former study was done for patients without history of diabetes, and diagnostic test during in hospitalization such as $\mathrm{HbA1C}$ and Oral glucose tolerance test were not analyzed [18].

So, this study aim was to investigate the correlation between admission blood glucose level and the no-reflow phenomenon in patients with AMI undergoing Primary PCI.

This study was cross-sectional study which included 280 patients presenting with acute ST segment elevation myocardial infarction who underwent primary PCI. Based on blood glucose level on admission, patients were classified into two groups: hyper glycemic group and euglycemic group.

As regard demographic data, males were more predominant $(73.2 \%)$ in this study than females $(26.8 \%)$ with mean age $(55.0 \pm$ 9.7) years. Among our patients, (49.6\%) had HTN, (39.3\%) had DM, (23.9\%) were dyslipidemic and (54.3\%) were smokers. Also, (20.4\%) had family history of CAD and (19.3\%) were known ischemic before.

Among our 280 patients, hyperglycemia group was 148, euglycemia group was 132. Age, HTN, dyslipidemia and CAD were higher among hyperglycemic group. Smokers were more in euglycemic group. Two groups showed statistical insignificant differences in all demographic data and risk factors except presence of diabetes mellitus which was 76 (51.4\%) in hyperglycemia group and 34 $(25.8 \%)$ in euglycemia group $(\mathrm{p}<0.001)$ which indicates perfect matching between groups.

This is similar to Kim et al. [19] in study about clinical impact of admission hyperglycemia on in-hospital mortality in acute myocardial infarction patients who reported that among 4,367 diabetics, hyperglycemia group was 2,270, non-hyperglycemia group was 2,097. Among 8,228 non-diabetics, hyperglycemia group was 665 and non-hyperglycemia group was 7,593. As the result of analyzing hyperglycemic, compared to nonhyperglycemic group, hyperglycemic group had higher percentage of hypertension, dyslipidemia, and non-ST segment elevation than euglycemic group. However, history of smoking was high in non-hyperglycemic group.

In this study, our patients had mean systolic blood pressure of (120.8 \pm 17.2$)$ mmhg and heart rate of $(91.4 \pm 13.9)$ bpm. Among both groups there were insignificant differences in systolic blood pressure and heart rate.

The present study patients had a mean onset of chest pain to admission time of $(8.5 \pm 5.8)$ minute with no significance difference between hyper and euglycemic groups.

Killip class $>2$ suggests that evidence of HF has been found [20]. Fajar et al. [21] showed that subjects with killip class $>2$ had 2.82 fold the risk for no reflow. The correlation between HF and no reflow is a complex involving neurohumoral activation that leads to imbalance between nitric oxide (NO) and reactive oxygen species (ROS). Reduced bioavailability of $\mathrm{NO}$ and abundant formation of ROS within vascular wall play an important role in endothelial dysfunction which is the basic of pre-existing micro vascular dysfunction mechanism [22].

In our study patients with Killip class III were (14.3\%) with statistically significant higher percentage in hyperglycemic group $(18.9 \%)$ than euglycemic group $(8.1 \%)(p=0.019)$.

Our laboratory findings found statistical significant elevation in creatinine in hyperglycemic group which was associated with the risk of no-reflow.

The correlation between creatinine level and no-reflow is complicated. Of the possible no-reflow mechanisms; the closest possible mechanism is through endothelial dysfunction. Although the association between renal function and endothelial dysfunction is unclearly elucidated. However, the basic correlation has been proposed. Creatinine level indicates renal function and elevated its level is associated with renal impairment [23].

On the other, reduction of renal function has been proven to cause retention of vasotoxic substances and cause metabolic changes that lead to increase ROS. These changes are believed to have an important role to create an atherogenic milieu [24].

As the result, plasma concentration of endothelium-derived protein will be increased and endothelium-dependent vasodilatation will be decreased. The changes of this level are responsible to increase soluble vascular cell adhesion molecule-1 (sVCAM-1) expression, the earlier step of endothelial dysfunction. Endothelial dysfunction also has a correlation with elevated blood glucose levels as reported in another study [25].

In addition, our laboratory findings also found statistical significant 
elevation in CK-MB in hyperglycemic group that was associated with the risk of no-reflow.

The correlation between CK level and the risk of no-reflow is possible through vascular contractility. CK, known as cardiac biomarker is an enzyme found primarily in the cardiac muscle and skeletal muscle and its elevation in serum is highly specific and sensitive for myocardial cell wall injury especially MI [26].

Our echocardiographic results found no significant difference between both groups. Similar to Kim et al. [19] who found no difference between groups in Echo data.

In our study, no-reflow (TIMI 0 and I) percentage was (34.3\%) as 38 (13.6\%) develop TIMI 0 and 58 (20.7\%) develop TIMI 1 but Reflow (TIMI II and III) was (65.7\%) as 22 (7.9\%) develop TIMI II and $162(57.8 \%)$ develop TIMI III with In-hospital mortality 36 (12.9\%). The incidence of no-reflow showed significant highest increase in diabetic hyperglycemic group 40 (52.6\%).

This is in agreement with Kim et al. [19] who found that Diabetics has significantly higher in-hospital mortality rate compared to non-diabetics. But in contrast to our study Kim et al. [19] found that, after classified into hyperglycemic and non-hyperglycemic, hyperglycemic in non-diabetics has significant no-reflow compared to diabetics.

In our study hyperglycemic group had statistical higher in hospital mortality rate, this is in accordance with other studies which demonstrated the relationship between admission hyperglycemia and short-term mortality in subjects without known DM after AMI [27].

In another study, findings highlight a 2-fold increase in mortality risk with hyperglycemia after STEMI, as an additive to clinical parameters [28].

Moreover, it is of interest to note that the probability of mortality was not modified when DM was added in our model, indicating that the predictive influence of DM was marginal. Similar results were found by Pinto et al. [27] in a subgroup of patients in CLARITY-TIMI 28 study trial.

The pathogenesis and risk factors of no-reflow are still incompletely understood. However, some literatures have pro-posed several mechanisms: pre-existing microvascular dys-function, distal microthrombo-embolization due to high platelet activity and much thrombus burden, ischemic injury, reperfusion injury, swelling of myocardial cells compressing microvascular vessels, and individual susceptibility [22,29].

To sum up, our study univariate factor analysis found a significant p-value for incidence of no-reflow as follow Age, DM, CK-MB and S. Creatinine. While multivariate after adjustment found that for incidence of no-reflow show significant $\mathrm{p}$-value in CK-MB and RBS.

Similar to another study which reported that age is widely known as one of risk factors for coronary heart disease (CHD). However, the understanding regarding age-related to no-reflow is limited.
This mechanism is probably through pre-existing micro vascular dysfunction. Advancing age is one of the major risk factors for cardiovascular disease because aging has the significant role in the development of vascular endothelial dysfunction and stiffening of large elastic arteries. Moreover, endothelial dysfunction has been known to impair coronary flow reserve (CFR) and increases the vulnerability of affected myocardium to the PCI induced injury [22].

In a recent study a total of 153 consecutive AMI patients undergoing primary PCI within 24 hours of symptom onset were included in this study. The participants were divided into normal flow group $(n=124)$ and slow/no-reflow group $(n=29)$ according to cineangiograms recorded during the period of PCI. Multivariate analysis revealed that $\mathrm{SI} \geq 0.66$, thrombus burden, and plasma glucose on admission were independent predictors for coronary slow/no-reflow [30].

In addition, diabetes mellitus; hypertension; and male have also been shown to have a correlation with endothelial dysfunction [25].

Smoking (OR 95\% CI=0.78 [0.65-0.94], p=0.0090), diabetes mellitus (OR 95\% CI=1.45 [1.16-1.81], p=0.0010), hypertension (OR 95\% CI=0.84 [0.76-0.93], $\mathrm{p}=0.0010$ ), symptom to reflow time (OR 95\% CI=1.92 [1.05-3.49], p=0.0330), killip class $>2$ (OR 95\% CI=2.82 [1.90-4.18], $\mathrm{p}<0.0001$ ) elevated blood glucose (OR 95\% CI=1.90 [1.22-2.95], p=0.0050), creatinine (OR 95\% $\mathrm{CI}=2.23[1.08-4.60], \mathrm{p}=0.0300)$, and peak $\mathrm{CK}(\mathrm{OR} 95 \% \mathrm{CI}=3.13$ [2.22-4.41], $\mathrm{p}<0.0001)$ were associated with the risk of no-reflow [21].

In contrast to us, Fajar et al. [21] found that elevated heart rate was associated with the risk of no-reflow. No study explains the direct correlation between heart rate and no reflow. Fajar et al. [21] proposed that HF may bridge the association between heart rate and no reflow. Although decreased HR variability was correlated with increased sympathetic or decreased vagal tone, which might predispose to ventricular fibrillation [31].

Also, Ndrepepa et al. [15] reported that univariable and multivariable logistic regressions were used to identify predictors of no reflow after primary PCI (see Methods section for variables entered into the models). In univariable analysis, 10 variables (age, smoking, previous MI, Killip class, serum creatinine, C-reactive protein, time-to-treatment interval, LVEF, baseline TIMI flow grade, and initial perfusion defect) were identified as predictors of no-reflow phenomenon. After application of backward variable selection method, 4 variables (previous MI, C-reactive protein, baseline TIMI flow grade, and initial perfusion defect) remained as significant independent predictors of no reflow after primary PCI.

In the current study, comparison between CK-MB $\geq 116 \mathrm{U} / \mathrm{L}$ and $\mathrm{RBS} \geq 241 \mathrm{mg} / \mathrm{dL}$ as a cut off for prediction of no-reflow as follow CK-MB had sensitivity of $60.4 \%$ and Specificity $56.5 \%$, while RBS had sensitivity of $72.9 \%$ and specificity of $56.5 \%$ (49.0-63.8). Similarly Dogan et al. [32] found that the prediction of "no-reflow" 
in patients with blood glucose level of $>225 \mathrm{mg} / \mathrm{dL}$ at admission $(\mathrm{OR}=5.125$; $95 \% \mathrm{CI}=2.128,12.347$; $\mathrm{p}<0.001)$.

In concordance with our current knowledge, the course of blood sugar level higher than the target value increased not only the mortality but also the risk of the development of "no-reflow" in patients of the study. It can be suggested that the effect of acute high blood sugar on the increase in mortality is related to the increased frequency of "no-reflow" in patients with acute myocardial infarction.

Another data that supports the current findings is the identification of the choice of $\mathrm{OAD}$ or else $\mathrm{OAD}+$ insulin treatment has no effect on the development of "no-reflow". According to this, high chronic blood sugar has no effect on the development of "no-reflow" but high acute blood sugar increases the risk. While chronic hyperglycemia is a risk factor for coronary artery disease, acute hyperglycemia is a risk factor for increased mortality in the course of MI [31].

Loss of sirtuin activity and NAD+levels with age are implicated in the etiology of a wide variety of cardiovascular and metabolic diseases, including atherosclerosis, endothelial dysfunction, acute cardiac syndromes, cardiomyopathy, hypertrophy and heart failure, arrhythmias, hypertension, metabolic syndrome, obesity and fatty liver, diabetes and dyslipidemia. The majority of these studies have been completed in animal models but human studies are increasing [33]

The sirtuin family of nicotinamide adenine dinucleotide (NAD+)dependent deacylases (SIRT1-7) is thought to be responsible, in large part, for the cardiometabolic benefits of lean diets and exercise and when up regulated can delay key aspects of aging [33].

Agnostics and programmed cell death with relevance to severity of global chronic disease progression. Proteomic profiles that include plasma Sirt 1 and protein analysis are critical to determine defects in the nuclear-mitochondria interaction relevant to the severity of cardiovascular disease, NAFLD, diabetes and neurodegenerative diseases. Sirt 1's control of biological systems in health and disease involve toxic amyloid beta and protein interactions with Sirt 1 repression associated with inactivation of drug/xenobiotic metabolism with acceleration of chronic disease progression. Interest in applying proteomics to disease progression requires early plasma Sirt 1 analysis for detection of disease protein biomarkers associated with inactivation rapid toxic amyloid beta and therapeutic drug metabolism [34].

\section{Limitations of the study}

Although all patients underwent coronary reperfusion within 90 min after blood glucose measurement at hospital admission, we do not know how the blood glucose level had changed before coronary reperfusion.

We also did not investigate how long hyperglycemia persisted before reperfusion. We did not perform the glucose tolerance test in all patients without a history of DM or a high $\mathrm{HbAlc}$ value.
However, several previous reports have demonstrated that $40 \%$ of patients with AMI, but without DM, had hyperglycemia on admission as in the present study.

We did not measure serum catecholamine levels, free fatty acids, blood viscosity, or red-cell deformability, which might be useful to clarify the relationship between the blood glucose level and infarct size. We could not successfully analyze the effects of the sulfonylurea agents, which might affect micro-vascular integrity, because of the small number of patients receiving these drugs.

\section{Conclusion}

To compare and analyze the effect of hyperglycemia on no-reflow phenomenon, in-hospital clinical outcome was investigated for diabetics and non-diabetics among Egyptian AMI patients based on status of admission hyperglycemia. From December 2018 to July 2019, 280 patients were enrolled and those were classified into 148 hyperglycemic and 132 euglycemic.

As the result, admission hyperglycemia with AMI among AMI patients was independent predictor for incidence of noreflow. Diabetics after classified into hyperglycemic and nonhyperglycemic, hyperglycemic in diabetics has significant high noreflow rate compared to non-diabetics.

\section{Conflicts of interest}

There are no conflicts of interest.

\section{References}

1. Sewdarsen M, Jialal I, Vythilingum S, et al. Stress hyperglycaemia is a predictor of abnormal glucose tolerance in Indian patients with acute myocardial infarction. Diabetes Res. 6(1): 47-49 (1987).

2. Oswald GA, Smith CC, Betteridge DJ, et al. Determinants and importance of stress hyperglycaemia in non-diabetic patients with myocardial infarction. BMJ. 293: 917-22 (1986)

3. Oswald GA, Yudkin JS. Hyperglycaemia following acute myocardial infarction the contribution of undiagnosed diabetes. Diabet Med. 4(1): 68-70 (1987).

4. Bolk J, Van-der Ploeg T, Cornel JH, et al. Impaired glucose metabolism predicts mortality after a myocardial infarction. Int J Cardiol. 79(3): 207-214 (2001)

5. Tansey MJ, Opie LH. Plasma glucose on admission to hospital as a metabolic index of the severity of acute myocardial infarction. Can J Cardiol. 2(6): 326331 (1986).

6. Malmberg K, Ryden L, Efendic S, et al. Randomized trial of insulin-glucose infusion followed by subcutaneous insulin treatment in diabetic patients with acute myocardial infarction (DIGAMI study): effects on mortality at 1 year. J Am Coll Cardiol. 26(1): 57-65 (1995)

7. Wahab NN, Cowden EA, Pearce NJ, et al. Is blood glucose an independent predictor of mortality in acute myocardial infarction in the thrombolytic era? J Am Coll Cardiol. 40(10): 1748-1754 (2002).

8. Norhammar A, Tenerz A, Nilsson G, et al. Glucose metabolism in patients with acute myocardial infarction and no previous diagnosis of diabetes mellitus: a prospective study. Lancet. 359(9324): 2140-2144 (2002).

9. Capes SE, Hunt D, Malmberg K, et al. Stress hyperglycaemia and increased risk of death after myocardial infarction in patients with and without diabetes: a systematic overview. Lancet. 355(9206): 773-778 (2000).

10. Malmberg K, Norhammar A, Wedel H, et al. Glycometabolic state at admission: Important risk marker of mortality in conventionally treated patients with diabetes mellitus and acute myocardial infarction: long-term 


\section{Research Article}

results from the Diabetes and Insulin-Glucose Infusion in Acute Myocardial Infarction (DIGAMI) study. Circulation. 99(20): 2626-2632 (1999).

11. Stranders I, Diamant M, Gelder VR, et al. Admission blood glucose level as risk indicator of death after myocardial infarction in patients with and without diabetes mellitus. Arch Intern Med. 164(9): 982-988 (2004).

12. Zeller M, Steg P, Ravisy J, et al. Prevalence and impact of metabolic syndrome on hospital outcomes in acute myocardial infarction. Arch Intern Med. 165(10): 1192-1198 (2005)

13. Oswald GA, Corcoran S, Yudkin JS, et al. Prevalence and risks of hyperglycaemia and undiagnosed diabetes in patients with acute myocardial infarction. Lancet. 323(8389): 1264-1267 (1984).

14. Thygesen K, Alpert JS, Jaffe AS, et al. Third universal definition of myocardial infarction. J Am Coll Cardiol. 60(16): 1581-1598 (2012).

15. Ndrepepa G, Tiroch K, Keta D, et al. Predictive factors and impact of no reflow after primary percutaneous coronary intervention in patients with acute myocardial infarction. Circ Cardiovasc Interv. 3(1): 27-33 (2010).

16. Squire IB, Nelson CP, Ng LL, et al. Prognostic value of admission blood glucose concentration and diabetes diagnosis on survival after acute myocardial infarction; results from 4702 index cases in routine practice. Clin Sci. 118(8): 527-35 (2010).

17. Kim EJ, Park OJ, Jeong MH, et al. Admission glucose is a useful prognostic factor in patients with acute myocardial infarction. Korean J Med. 79(1): 2331 (2010).

18. Tabit CE, Chung WB, Hamburg NM, et al. Endothelial dysfunction in diabetes mellitus: molecular mechanisms and clinical implications. Rev Endocr Metab Disord. 11: 61-74 (2010).

19. Kim EJ, Jeong MH, Kim JH, et al. Clinical impact of admission hyperglycemia on in-hospital mortality in acute myocardial infarction patients. Int J Cardiol. 236(1): 9-15 (2017). 区

20. Talley NJ, Connor OS. Clinical Examination: A Systematic Guide to Physical Diagnosis. 7th ed. Chatswood: Elsevier Australia CAN. (2014).

21. Fajar JK, Heriansyah T, Rohman MS, et al. The predictors of no reflow phenomenon after percutaneous coronary intervention in patients with ST elevation myocardial infarction: A meta-analysis. Indian Heart J. 70(3): S406-S418 (2018).凶
22. Gupta S, Gupta MM. No reflow phenomenon in percutaneous coronary interventions in ST-segment elevation myocardial infarction. Indian Heart J. 68(4): 539-551 (2016)

23. Gelfand EV, Cannon CP. Management of Acute Coronary Syndromes. West sussex: John Wiley \& Sons Ltd. Arch Intern Med. 164: 982-988 (2009).

24. Topol EJ, Teirstein PS. Textbook of Interventional Cardiology. 7th ed. Philadelphia: Saunders, (2015).

25. Koller A. Perspectives: microvascular endothelial dysfunction and gender. Eur Heart J. 16(1): 16-19 (2014).

26. Mythili S, Malathi N. Diagnostic markers of acute myocardial infarction. Biomed Rep. 3(1): 743-748 (2015).

27. Pinto DS, Kirtane AJ, Pride YB, et al. Association of blood glucose with angiographic and clinical outcomes among patients with ST-segment elevation myocardial infarction (From the CLARITY-TIMI-28 Study). Am J Cardiol. 101(3): 303-307 (2008).

28. Sanjuán R, Núńez J, Blasco ML, et al. Prognostic implications of stress hyperglycemia in acute ST elevation myocardial infarction. Prospective observational study. Rev Esp Cardiol. 64(3): 201-207 (2011).凹

29. Wong DT, Puri R, Richardson JD, et al. Myocardial 'no-reflow'-diagnosis, pathophysiology and treatment. Int J Cardiol. 167(5): 1798-1806 (2013).

30. Wang Q, Shen H, Mao H, et al. Shock Index on Admission Is Associated with Coronary Slow/No Reflow in Patients with Acute Myocardial Infarction Undergoing Emergent Percutaneous Coronary Intervention. Int J Cardiol. (2019).

31. Hasenfuss G. Benefit of heart rate reduction in heart failure. Curr Heart Fail Rep. 7: 156-158 (2010).

32. Dogan NB, Ozpelit E, Akdeniz, S, et al. Simple clinical risk score for noreflow prediction in patients undergoing primary Percutaneous Coronary Intervention with acute STEMI. J Med Sci. 31(3): 576-581 (2015).

33. Kane AE, David A. Sinclair Sirtuins and NAD+ in the Development and Treatment of Metabolic and Cardiovascular Disease. Circ Res. 123(7): 868885 (2018).

34. Martins IJ. Sirtuin 1, a Diagnostic Protein Marker and its Relevance to Chronic Disease and Therapeutic Drug Interventions. Ecrinocorns. 6. 209-215 (2018). 